\title{
Abyssinones and related flavonoids as potential steroidogenesis modulators
}

\author{
Kaushik S. Hatti, Latha Diwakar, G. Venkateswara Rao, Anil Kush, G. Chandrasekara Reddy* \\ Vittal Mallya Scientific Research Foundation, PB No 406, K.R. Road; Bangalore 560004, India, G. Chandrasekera Reddy - Email: \\ gcreddy@vmsrf.org; * Corresponding author \\ Received February 19, 2009; revised April 8, 2009; accepted May 11, 2009; published June 28, 2009
}

Abstract:

Abyssinones and related flavonoids were screened against 3 enzymes (3ßHSD, 17 $\beta$ HSD and Aromatase) of steroidogenesis pathway. The virtual screening experiment shows high affinity for flavonones than their respective chalcones. A 4' $-\mathrm{OH}$ blocked prenylated flavonone $\mathbf{2 b}$ (2-(2', 2'-dimethyl chroman-6'-yl)-7-hydroxy chroman-4-one) had consistent binding affinity to all the three enzymes used in this study showing higher binding affinity to aromatase. A good correlation was observed between cytotoxic data (MCF-7, breast cancer cell line) and docking results indicating flavonone as a better steroidogenesis modulator in hormone dependent cancer.

Keywords: flavonoids; steoidogenesis modulators; docking; model; cancer

\section{Background:}

Cancer is one of the major causes of death across the world. It is largely controllable disease and highly susceptible to modulation by dietary factors. Epidemiologic evidence suggests an association between the consumption of fruits and vegetables with reduced incidence of cancer [1, 2]. Over the years, numerous bioactive compounds have been identified that contribute to these beneficial health effects. Polyphenols are the most abundant antioxidants in human diet and are widespread constituents of fruits, vegetables, cereals and in beverages such as tea, coffee and wine. Polyphenols are gaining importance because of their various biological activities such as anti-cancer [3], anti-viral [4], anti-inflammatory [5], anti-ulcer [6] and anti-fungal [7] along with their antioxidant properties. As antioxidants, polyphenols may protect cell constituents against oxidative damage. Therefore, they can limit the risk of various degenerative diseases associated with oxidative stress, such as cardiovascular diseases, diabetes and cancer [2, 8]. In complex biological systems, polyphenols exhibit several additional properties which may be independent of conventional antioxidant activities leading to novel mechanism of interacting with cell signaling and modulating several cell activities [9 10].

Flavonoids are polyphenolic compounds which are fundamental components of the human diet present as constituents of flowering plants, particularly of food plants. They are phenyl substituted chromones (benzopyran derivatives) consisting of a 15-carbon basic skeleton (C6C3-C6). Several plants and spices containing flavonoid derivatives have found application as disease preventive and therapeutic agents in traditional medicine in Asia for thousands of years [11]. Epidemiological investigations and human clinical trials indicate that flavonoids have important effects on cancer chemoprevention and chemotherapy. Many mechanisms of action such as antiproliferation, cell cycle arrest, induction of apoptosis and differentiation, inhibition of angiogenesis, reduction of oxidative damage and reversal of multidrug resistance or a combination of these mechanisms have been identified [12]. Most of the biological activities are attributed to substitution of prenyl and hydroxyl groups present in the flavonoid ring system [13].

Breast cancer is the leading cause of cancer in women worldwide, both in the developed and developing countries. The breast cancer ranked second after cervix in the developing countries [14]. It is well established that estrogen modulates cell function. Studies have shown that cell proliferation in breast cancer depends on levels of estrogen and estrogen receptors especially in hormone dependent breast cancer [15]. Hence the enzymes involved in synthesis and regulation of estrogen becomes important targets for breast cancer therapy. In steroidogenesis, estrogen is synthesized from cholesterol as starting molecule to androstenedione, a compound with androgenic activity and finally converted into estrodiol or estrone by regulation of three important enzymes, $3 \beta \mathrm{HSD}, 17 \beta \mathrm{HSD}$ and Aromatase. Amongst all, aromatase - an enzyme of the cytochrome P450 family is a very well considered and important pharmacological target, particularly for the treatment of breast cancer [16]. Natural polyphenolic compounds such as flavones, flavonones, resveratrol, and oleuropein are shown to be strong aromatase inhibitors [17], but inhibit to a lesser extent the other two enzymes $3 \beta H S D$ and $17 \beta H S D[18,19]$. Abyssinones are prenylated flavonoids isolated from plant Erythrina abyssinica. These molecules have gained attention since abyssinone II [20] reported to show aromatase inhibitory activity. We have recently synthesized Abyssinone-I (2a), abyssinone-II (2c) and related compounds and studied their anti-oxidant properties [21].

In the present study, we have screened a set of flavonones and their respective chalcones (Figure 1) including abyssinones for the cytotoxic effect in MCF-7 (breast cancer) cell line. As these flavonoids are reported to be effective aromatase inhibitors, docking studies were carried out to screen potent molecules with high cytotoxic effect as well as a good inhibition for the $3 \beta \mathrm{HSD}$ (E.C.1.1.1.145), 17 BHSD (E.C.1.1.1.62) and/or aromatase (E.C.1.14.14.1) enzymes, since aromatase inhibitors are target for new drug synthesis to treat hormone-dependent cancers, in addition to breast cancer [22]. 


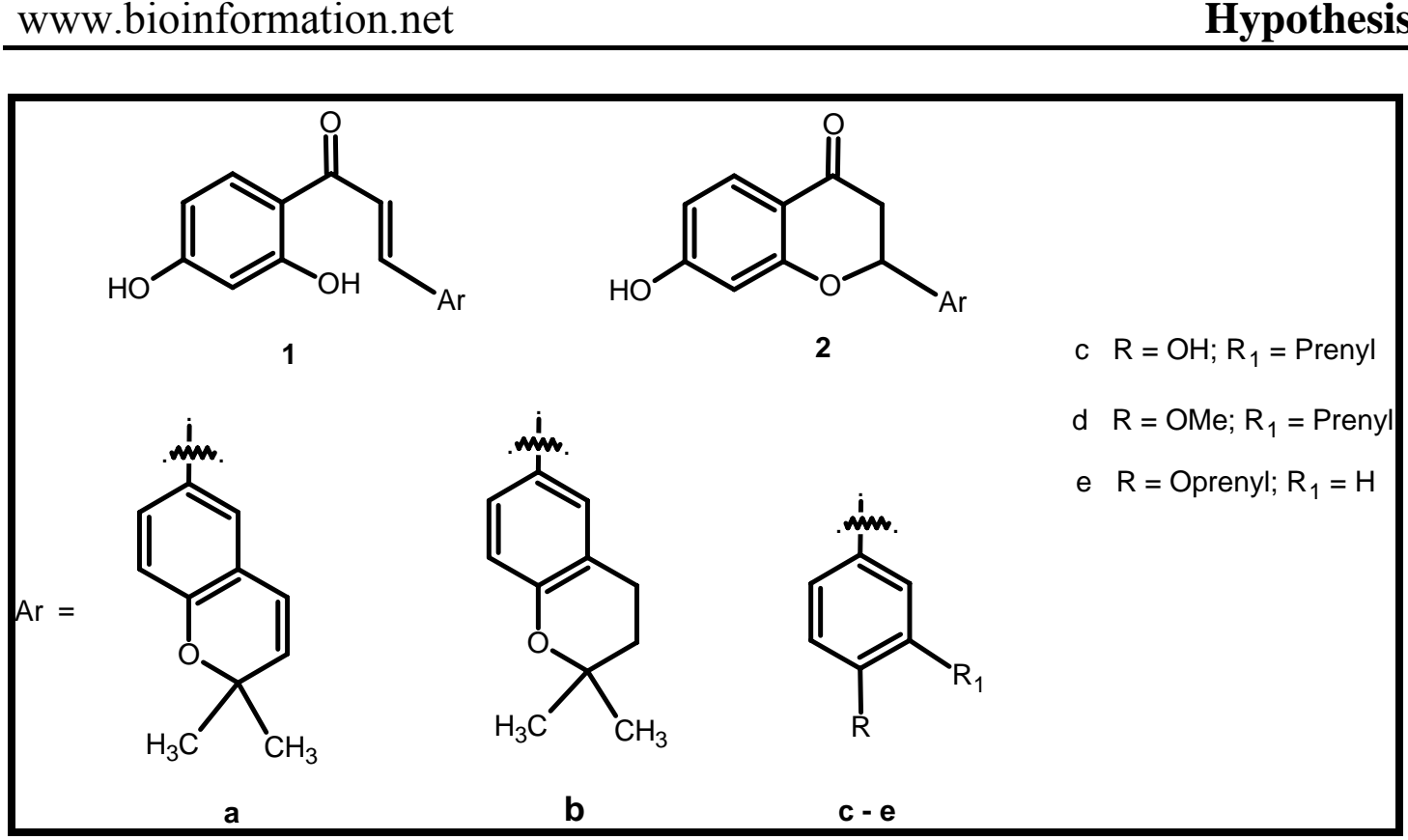

Figure 1: Compounds under study.

\section{Methodology:}

\section{Chemicals:}

MTT [3-(4, 5- dimethyl-thiazol-2-yl)-2, 5-diphenyl -2Htetrazolium bromide], Dimethyl sulfoxide, DMEM (D5648) medium from Sigma-Aldrich, Heat inactivated serum from Gibco BRL, Antibiotic \& antimycotic solution and other chemicals were purchased from Himedia, India.

\section{Cell culture:}

MCF-7 human breast carcinoma cell line (Purchased from NCCS, Pune, India) was maintained in DMEM medium supplemented with $10 \%$ FBS and antibiotics solution at $37^{\circ} \mathrm{C}$ in ambient air with $5 \% \mathrm{CO}_{2}$. The sub culturing was done every 2-3 days. The flavonoids were dissolved in dimethyl sulfoxide with serial dilution ranging from $2.5 \mu \mathrm{M}-100 \mu \mathrm{M}$. MTT assay: Quantification of cell viability was performed using the cleavage of MTT to a blue colored complex (formazan), which is indicator for mitochondrial succinate dehydrogenase activity in viable cells [23]. Cells were seeded in 96 well plates, supplemented with flavonoids of different concentration and incubated for $24 \mathrm{hrs}$. After incubation $20 \mu \mathrm{l}$ of $5 \mathrm{mg} / \mathrm{ml}$ MTT dissolved in PBS was added to culture well and further incubated for $4 \mathrm{hrs}$ at $37^{\circ} \mathrm{C}$. Supernatants were removed and $200 \mu \mathrm{l}$ of DMSO was added to each well. The optical density was measured at $580 \mathrm{~nm}$ with molecular Devices ELISA reader. Dose response curve were plotted after converting the mean values in to percentages of control response. Flavonoid doses resulting in 50\% inhibition of live cells were derived from plotted data.

\section{In-silco analysis:}

\section{Preparation of proteins}

The Comparative Modeled structure of cytochrome P450 aromatase (Pdb code: 1TQA), modeled structure of 3 $\beta H S D$ [24] and crystal structure of $17 \beta \mathrm{HSD}$ (Pdb code: 1FDT) was considered for docking studies. All the protein structures were prepared for docking by adding polar hydrogen, except haem all hetero atoms removed and all the bonds set as rigid using MGLTools 1.5.1.

\section{Preparation of ligands}

All the ligands were drawn; geometrically optimized using $\mathrm{ACD} /$ ChemSketch 10.0 and energy minimized using The Dundee PRODRG2 Server [25]. All the maximum allowed torsions in the ligands were set as flexible.

\section{Docking studies using AutoDock 4.0}

Using MGLTools 1.5.1, a grid spacing of $0.374 \AA$ with $126 \times 126 \times 126$ points for all the proteins were prepared. Docking for 100 number of GA runs was carried out using Lamarckian Genetic Algorithm (LGA) and all other parameters set to default. The top ranked model in the lowest energy cluster with maximum cluster size was considered for all further interaction studies.

\section{Discussion:}

It is already known that flavonoids are steroidogenesis modulators [18] and also have been widely exploited as inhibitors of aromatase which is a regulatory enzyme in the synthesis of estrogen [17]. Out of ten prenylated compounds screened for cytotoxic activity in MCF cellline, compound 1c showed the lowest IC50, 2e being least cytotoxic, while rest showed IC50 values varying from 60 to $70 \mu \mathrm{M}$ (Figure 2). Chalcones had higher cytotoxic effect and their binding affinity with steriodogenic enzymes was less when compared to flavonones. Though, 1c had good cytotoxic effect, its binding affinity for steroidogenesis enzymes considered in the present study was least. Different substitutants on flavonones influence the binding affinity of the compounds to the enzymes. Presence of prenyl group in flavonone enhances its binding affinity to $3 \beta \mathrm{HSD}$ and $17 \beta \mathrm{HSD}$ while there was minimal influence on aromatase binding. 


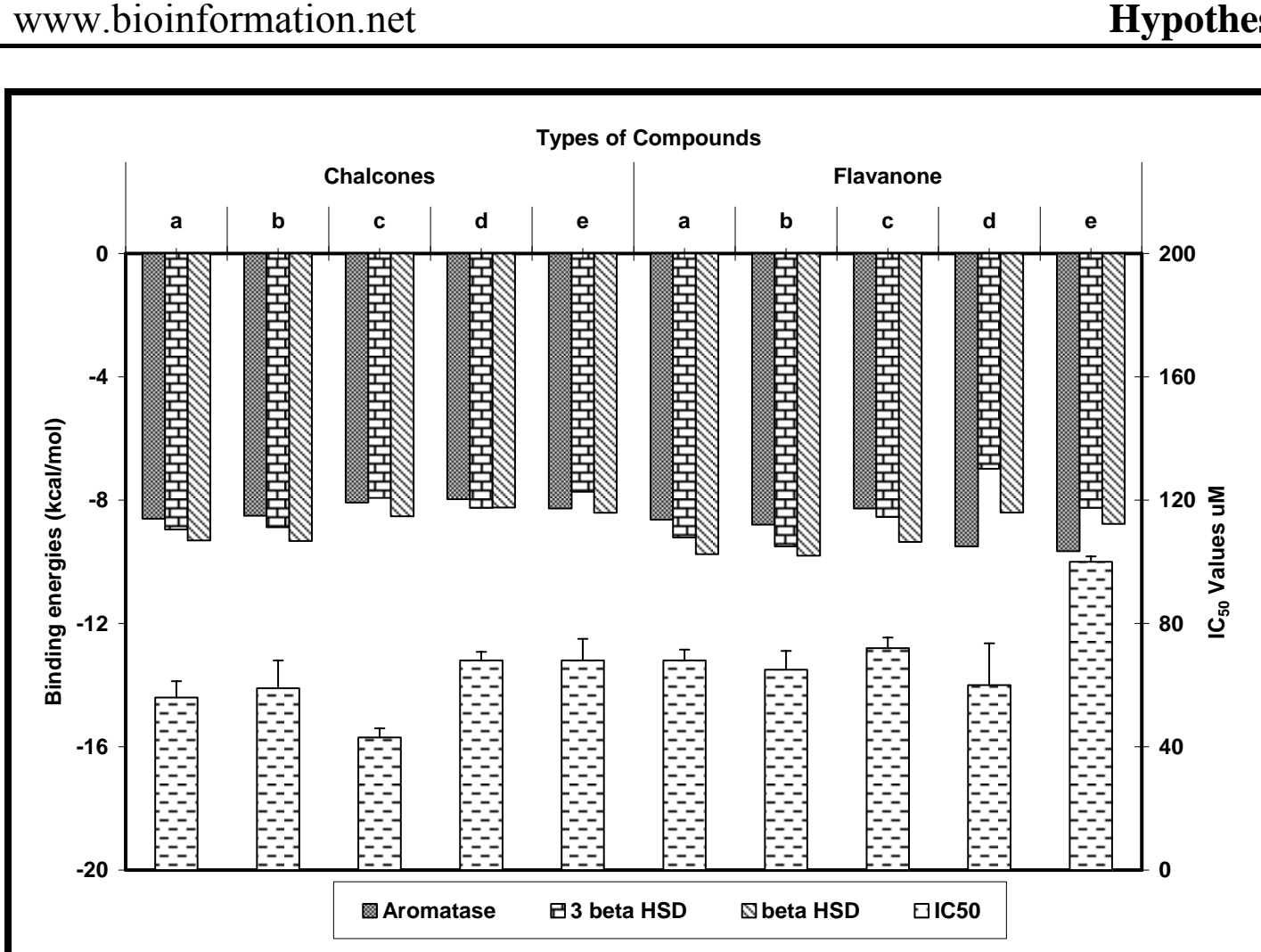

Figure 2: Graph plot of Binding energies of all the compounds with their respective IC50 values.

\section{Aromatase:}

Though natural ligand, androstenedione did not show any H-bonds in our docking studies, it showed electrostatic interactions with Val370. Whereas, 7-hydroxy in flavonones forms H-bonds with Val370 showing competitive affinity to the same amino acid over natural ligand. The present study reveals that, flavonones with protected 4' hydroxyl showed better aromatase inhibitory activity than their free hydroxyl analogs irrespective of the presence of prenyl groups. Present docking data matches well with the aromatase inhibitory assay results reported by Moriaty et al [26].

\section{BHSD:}

Our study showed that, 3, 17 hydroxyls of androstenediol forms hydrogen bonds with Ser221 and Ile192 respectively. We have also observed that in case of Abyssinone I (2a) and its analog (2b) carbonyl and oxygen in 4' position formed hydrogen bonds with $\operatorname{Arg} 198$ and Ser221 respectively. As Ser221 forms hydrogen bond in both the cases, the interaction might be contributing factor to the binding affinity of the compounds. The common electrostatic interactions found between the enzyme and flavonones 2a, 2b and natural ligand are Val223, Tyr193, Lys219, Phe199, Ile192, Ser221, Asn318 and Tyr191.

\section{$17 \beta H S D:$}

We found that, 3-hydroxy of Estrone formed hydrogen bond with Glu194. Whereas, in case of $2 \mathrm{a}$ and $2 \mathrm{~b} 7$ hydroxy and 4-carbonyl formed hydrogen bond with Gly144 and Val188 respectively. Ser142, Pro187, Val188, Phe226 and Gly186 amino acids of the enzyme were commonly interacting with $2 \mathrm{a}, 2 \mathrm{~b}$ and natural ligand considered.

\section{Conclusion:}

Steroidogenesis enzymes (aromatase, 17ßHSD and $3 \beta \mathrm{HSD}$ ) which are potential target of hormone dependent cancer were considered for docking and screening studies. This work finds application using these targets to design drugs for the treatment of hormone dependent cancer. The competitive binding efficiency of enzyme targets with prenylated and non-prenylated flavanoids confers them as steroidogenic modulator. Consequently, the approach is useful in designing novel molecules for treatment of hormone dependent cancer.

\section{Reference:}

[1] TM de Kok et al., Eur J Nutr. (2008) 47, 51-59. [PMID:18458834]

[2] M Kampa et al., Rev Physiol Biochem Pharmacol. (2007)159, 79-113. [PMID:17551696]

[3] HM Cidade et al., Planta Med. (2001) 867-70. [PMID: 11745028]

[4] KM Meragelman et al., J Nat Prod. (2001) 64:5468. [PMID:11325248]

[5] DS Jang et al, Phytochemistry (2002) 61(7):867-72 [PMID:12453581]

[6] J Yamahara et al., Chem Pharm Bull (1990) 38(4):1039-44 [PMID:2379277]

[7] S Tahara et. al., Agric. Biol. Chem. (1984) 48, 1471-1477.

[8] S. Ramos, J Nutr Biochem. (2007) 18, 427-442. [PMID:17321735] 
[9] J Mojzis et al., Pharmacol Res. (2008) 57, 259-265 [PMID:18387817]

[10] S Ramos et al., Mol Nutr Food Res. (2008) 52, 507 526. [PMID:18435439]

[11] C Kandaswami et al., In Vivo. (2005) 19, 895-909. [PMID:16097445]

[12] E Rudolf et al., Anticancer Agents Med Chem (2007) 5, 559-575. [PMID:17896916]

[13] RJ Rodriguez et al., Food and Chemical Toxicol. (2001) 39, 437-445. [PMID:11313109]

[14] PN Notani Current Science. (2001) 81, 465.

[15] HO Lee \& YY Sheen Arch Pharm Res. (1997) 20, 566-571. [PMID:18982261]

[16] F Rojo et al., Semin Diagn Pathol (2008) 25, 245 261.

[17] MA Neves et al., Chem Med Chem (2007) 12, 1750-1762. [PMID:17910019]

[18] S Ohno et al., J. Stero. Biochem. Molbio (2002) 80, 355-363. [PMID:11948020]
[19] P Brozic et. al., Mol Cell Endocrinol. (2008) Sep 17. [PMID:18835421]

[20] A Maiti et al., J Med Chem (2007) 50, 2799-2806. [PMID:17511439]

[21] G.V. Rao et al., Europ. J. Med. Chem. (2009) 44, 2239-45. PMID: 18603336

[22] R Le Lain et al., J Enzyme Inhib. (2001) 16, 35-45. [PMID:11496833]

[23] A Visconti et. al., Mycopathologica. (1991) 113, 181-186. [PMID: 2067563]

[24] U Pieper et al., Nucleic Acids Research. (2006) 34, D291-D295. [PMID:16381869]

[25] A. W. Schuettelkopf \& D. M. F. van Aalten. Acta Crystallogr D Biol Crystallogr. (2004) D60, 135563. [PMID:15272157]

[26] RM Moriarty et. al., Eur. J. Med. Chem. (2006) 41, 263-267. [PMID:16330130]

Edited by P. Kangueane

Citation: Hatti et al, Bioinformation 3(9): 399-402 (2009) License statement: This is an open-access article, which permits unrestricted use, distribution, and reproduction in any medium, for non-commercial purposes, provided the original author and source are credited. 